\title{
Experimental Results for Outpatient treatment with Lu-177 Dotatate External Dosimetry
}

\author{
Mushref Algarni ${ }^{* 1,2}$, Robert Stodilka ${ }^{1}$, Janine Riffel ${ }^{1}$, David Laidley ${ }^{1}$ \\ ${ }^{1}$ Department of Nuclear Medicine, University Hospital, Canada \\ ${ }^{2}$ Department of Diagnostic Medical Imaging and Nuclear Medicine, King Fahd Military Medical Complex, Saudi Arabia
}

Received: February 11, 2018; Published: February 23, 2018

*Corresponding author: Mushref Algarni, Department of Nuclear Medicine, London Health science, University Hospital, Schulich School of Medicine \& Dentistry, Western University London, Ontario, Canada, Email: fresh20033@hotmail.com

\section{Introduction}

London health sciences center is a frequent user of Lu-177 Dotatate for the treatment of neuroendocrine disease. We began using this radionuclide therapy as an in-patient procedure, where patients were released at 20 hours' post therapy administration with minimal restrictions. Over the course of 2013, we worked to transform this therapy into an outpatient procedure, where patients were released at 4-6 hours' post therapy administration with major restrictions. The previous methods and data we presented in December 17, 2013 were based on dose rates derived from cumulated doses measured over approximately 16 hours, and an assumption of Lu-177 clearance based on radioactive decay only. Since 2013, our hospital has gained more experience with Lu-177 Dotatate, enabling us to develop a better understanding of dosimetry. We describe new dose measurements, a new model we developed to describe our observations, and a revised schedule of patient release and restriction duration. Compared against previous measurements from 2013, our new measurements are of instantaneous (not cumulative) dose rates, and we now consider Lu-177 clearance to proceed both by physical decay and biologic excretion. Our new proposed model combines our experimental results with results from literature. The model will state the dose rate from time of discharge can be modeled as a decaying double exponential function [1-5].

\section{Proposed Model}

\section{Clearance}

Clearance processes are important to understand because they will allow us to predict dose rate from a radionuclide therapy patient after the patient is released from the hospital. Clearance kinetics for many radiopharmaceuticals can be described as having an early phase and a late phase. Early phase clearance is often dominated by protocol- and patient-specific variables that influence nonspecific uptake (for example: patient hydration, renal function). Conversely, late-phase clearance is usually from disease-specific uptake (for example: from a tumor). Many authors in literature model clearance kinetics using first-order approximations (first order rate kinetics), resulting in clearance equations with decaying exponential functions [6-10]. Following these examples, we propose the clearance of our radiotherapy product can be modeled as a summation of two exponential decay functions: [Equation 1]

$$
C L R(t)=\alpha * \exp \left(-\lambda_{1} * t\right)+\beta * \exp \left(-\lambda_{2} * t\right)
$$

Where $\lambda_{1}$ and $\lambda_{2}$ describe the early phase and late phase effective decay constants, respectively; and $\alpha$ and $\beta$ represent the respective proportions of our radiotherapy product governed by the early and late phase clearance processes. Note that the relationship between decay constant and half-life is $\lambda=\ln (2) / \mathrm{t} 1 / 2$ where $t 1 / 2$ is half-life (Figure 1).

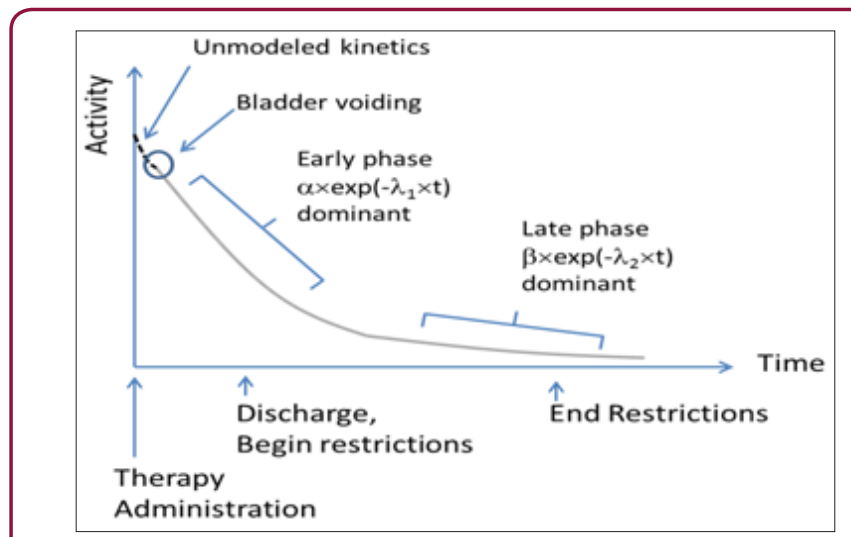

Figure 1: In this figure, the radionuclide therapy is administered at time $=0$. At some point, the patient first voids their bladder, after which we begin to model the therapy product's clearance kinetics. At first, clearance is rapid; and eventually clearance slows.

\section{Dosimetry}

The total estimated effective dose (EEDTOTAL) received by an individual in close proximity to the patient is proportional 
to the area under the patient activity vs time curve, from [time = discharge] to [time $=$ infinity] weighted by patient restrictions. We express this as follows: [Equation 2]

$$
E E D_{\text {Total }}=E E D_{R}(D S \rightarrow R E)+E E D_{u}(\mathrm{R} E \rightarrow \infty)+D_{i}
$$

Where $D_{S}=$ discharge time and $R_{E}=$ restrictions end time. $E E D_{R}$ is the estimated effective dose received by the caregiver from discharge to end of restrictions, during which restrictions are in effect. We define it as: [Equation 3]

$$
E E D_{R}(D S \rightarrow T R)=\frac{R V T Q_{0}}{r^{2}} E_{R} \int_{D S}^{T R} C L R(t) d
$$

Where,

$\mathrm{R}$ [unitless] = dose reduction factor due to patient self-shielding (attenuation);

$\mathrm{V}$ [unitless] = dose reduction factor due to patient voiding after therapy administration;

$\mathbb{Q}\left[\mathrm{uSv} \mathrm{m}^{2} / \mathrm{MBqhr}\right]=$ specific gamma constant for Lu-177;

$\mathrm{Q}_{0}[\mathrm{MBq}]=$ initial amount of Lu-177 administered to patient;

$\mathrm{r}$ [meters] = distance between patient and exposed individual; $\mathrm{E}_{\mathrm{R}}$ [unitless] = restricted occupancy factor; and $\mathrm{t}$ [hours $]=$ time.
$\mathrm{EED}_{\mathrm{U}}$ is defined the same as $\mathrm{EED}_{\mathrm{R}^{\prime}}$ except the restricted occupancy factor, $E_{R}$, is replaced with the unrestricted occupancy factor, $\mathrm{E}_{\mathrm{U}}$; and the limits of integration are [time $\left.=\mathrm{RE}\right]$ to $[$ time $=$ infinity]. Integrating Equation 2 is straight-forward, but leads to a lengthy equation that we omit here for brevity [10-15]. The term $D_{i}$ accounts for dose to the caregiver arising from internalized radionuclide's from the released patient, after NRC Regulatory guide 8.39. This is expressed as:

$$
D_{i}=e-i n(2) D S / t_{1 / 2} \cdot Q_{0} \cdot\left(10^{-5}\right) . D C F
$$

Where $\mathrm{t} 1 / 2$ is the Lu-177 physical half-life, $10-5$ is the assumed fractional intake, and DCF is the dose conversion factor, taken as 0.05 [uSv/MBq]. What remains now is to assign numerical values to the variables. We have conducted an experiment to measure some of these values; whereas others are taken from literature, as will be described next.

Objective 2: Calculation of Voiding (V): We determined the fraction of radiotherapy product in the patient after voiding by comparing pre- and post-void dose-rate measurements in Table 1. We found the average ratio to be 0.87 [15-20]. The corresponding value in literature is 0.54 . This void ratio is highly dependent on protocol design and time of voiding. All of our patients were instructed to void 1 hour post-administration of therapy.

\begin{tabular}{|c|c|c|c|c|c|}
\hline Patient & Activity [MBq] & $1 \mathrm{hr}$ pre-void [uSv/hr & $1 \mathrm{hr}$ post-void [uSv/hr & $5 \mathrm{hrs}[\mathrm{uSv} / \mathrm{hr}]$ & $20 \mathrm{hrs}[\mathrm{uSv} / \mathrm{hr}$ \\
\hline 1 & 5500 & 6.3 & $\mathrm{n} / \mathrm{a}$ & 2.5 & 1.2 \\
\hline 2 & 3700 & $\mathrm{n} / \mathrm{a}$ & 4.3 & 2.2 & 1.1 \\
\hline 3 & 5500 & 6.5 & $\mathrm{n} / \mathrm{a}$ & 2.7 & 1.2 \\
\hline 4 & 5500 & 5.2 & 4.7 & 1.9 & 0.8 \\
\hline 5 & 3700 & 5.3 & 4.8 & 2.7 & 1.1 \\
\hline 6 & 3700 & 4.8 & 3.9 & 1.7 & 0.8 \\
\hline
\end{tabular}

Table 1: Calculation of Voiding (V) we determined the fraction of radiotherapy product in the patient after voiding by comparing pre- and post-void dose-rate measurements.

Objective 3: Parameters for Clearance Model CLR( $t)$ : The following values for CLR $(\mathrm{t})$ parameters (Table 2). For interest, we note that the effective half-life associated with $\lambda_{1}$ is $t_{1 / 2}=2.1$ [hours], and $\lambda_{2}$ is $_{1 / 2}=91$ [hours].

Table 2: CLR (t) parameters

\begin{tabular}{|c|c|}
\hline Parameter & Value \\
\hline$\otimes$ & 1.12 \\
\hline$\square$ & 0.269 \\
\hline$\nabla$ & 0.328 \\
\hline$\square$ & $7.63 \times 10^{-3}$ \\
\hline
\end{tabular}

Checking Model again Measurements: As a check of our results so far, we can plot our predicted dose rate for patients as a function of time; and compare this prediction with our measurements. Our predicted dose rate is given by:

$$
\text { PredicatedDoseRate }[u S V]=\frac{R V T Q_{0}}{r^{2}}\left[\alpha * \exp \left(-\lambda_{1} * t\right)+\beta * \exp \left(-\lambda_{2} * t\right)\right]
$$

This predicted dose rate is compared with experimental measurements in Figure 2. For the case of 3700 MBq administered activities.

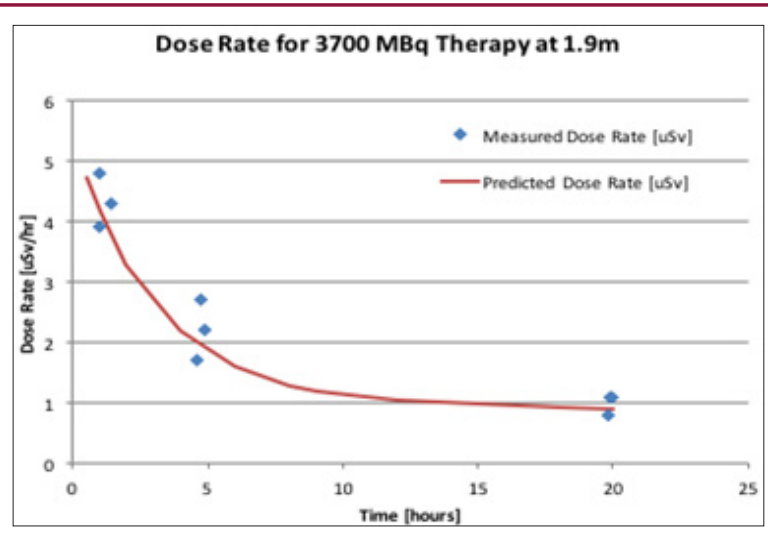

Figure 2: Dose rate for $3700 \mathrm{MBq}$ Therapy at $1.9 \mathrm{~m}$.

\section{Model-Based Predictions of EED}

In all cases, restrictions are assumed to result in 0.25 occupancy (Table 3).

\section{Correlation with December 172013 Data}

In our 2013 correspondence to you, we described an experiment where we measured cumulative doses from 14 patients. 
Dosimeters were placed in various locations in patients' rooms and measured over different time periods (up to 20 hours' post therapy administration). During this time, patients moved around the room: Table 3: Model-Based Predictions of Eed. from their bed to the bathroom, for example. We can compare this previous experimental data with our current model as follows:

\begin{tabular}{|c|c|c|c|c|c|c|}
\hline Scenario & Activity [MBq] & Discharge (DS) [hours] & Restriction End (RE) [hours] & $\mathbf{E E D}_{\mathrm{R}}[\mathrm{uSv}]$ & $\mathbf{E E D}_{\mathrm{u}}[\mathrm{uSv}]$ & $\mathbf{E E D}_{\text {TOTAL }}[\mathbf{u S v}]$ \\
\hline 1 & 3700 & 6 & 6 & 0 & 478 & 478 \\
\hline 2 & 3700 & 6 & 22 & 15.2 & 418 & 433 \\
\hline 3 & 5500 & 6 & 6 & 0 & 711 & 711 \\
\hline 4 & 5500 & 6 & 22 & 22.6 & 621 & 643 \\
\hline 5 & 5500 & 22 & 22 & 0 & 621 & 621 \\
\hline 6 & 7400 & 6 & 22 & 30.4 & 835 & 866 \\
\hline 7 & 7400 & 22 & 22 & 0 & 835 & 835 \\
\hline
\end{tabular}

a) In our previous study, the average activity administered was $5500 \mathrm{MBq}$, and the maximum was $7400 \mathrm{MBq}$.

b) We consider the longest cumulative dose integration time window in order to average patient movement: 4- 20 hours post therapy administration.

Table 4: Comparison of our 2013 data with our current mode. The corresponding calculations using our proposed model.

\begin{tabular}{|c|c|c|c|c|}
\hline Scenario & Activity [MBq] & 2013 Measurements [uSv] & Proposed Model [uSv] & Difference between measurement vs model [\%] \\
\hline 8 & 5500 & 78 (average of all patients) & 101 & $22 \%$ \\
\hline 9 & 7400 & 180 (patient 12) & 136 & $32 \%$ \\
\hline 9 & 7400 & 140 (patient 14) & 136 & $3 \%$ \\
\hline
\end{tabular}

The same parameters can be entered into our model: an activity of 5500 or $7400 \mathrm{MBq}$, and a distance of 1 meter. We can use EEDR if we set $D S=4$ and $R E=20$, and restriction occupancy fraction to 1 [20-25] (Table 4).

\section{Scenario 8}

From our 2013 data, the average dose measurement was $78 \mathrm{uSv}$ (assuming the average activity of $5500 \mathrm{MBq}$ ). Our proposed model, assuming $5500 \mathrm{MBq}$, yields a dose of $101 \mathrm{uSv}$.

\section{Scenario 9}

From our 2013 data, we have $0.18 \mathrm{uSv}$ as a maximum dose (with $7400 \mathrm{MBq}$ administered activity), and $140 \mathrm{uSv}$ as the second highest recorded dose. Our model predicted 136 uSv. Our 2013 data shows variability, and there are differences compared with our proposed model ranging from $3 \%$ to $32 \%$. Part of the variability and differences may be due to the patients of 2013 moving around their hospital rooms (their precise movement patterns over 20 hours were not monitored). However, overall we consider the 2013 experiment results and our model predictions reasonably close, especially considering we used two very different methods to arrive at dose. Our intention is to eventually use our new model to guide decisions for Lu-177 Dotatate patient restrictions and release from our institution. We would appreciate your thoughts on our proposal [25-28].

\section{References}

1. Olmstead C (2015) Quantifying public radiation exposure related to lutetium-177 octreotate therapy for the development of a safe outpatient treatment protocol. Nucl Med Commun 36: 129-134. c) The dosimetry location we will select is the bottom of the patients' beds (near patient feet).

d) We will take this location as approximately "1 meter" away from the patient.

2. Calais PJ, Turneur JH (2014) Radiation Safety of outpatient $177 \mathrm{Lu}-$ octrotate radio peptide therapy of neuroendocrine tumors. Ann Nucl Med 28: 531-539.

3. Fitschen J (2011) External radiation exposure and effective half-life in Lu-177-Dota-Tate therapy Z. Med Phys 21(4): 266-273.

4. (1997) Regulatory Guide 8.39 Release of Patients Administered Radioactive Materials April 1997 United States Nuclear Regulatory Commission, USA.

5. Seregni E (2010) Treatment with tandem [90Y] DOTA-TATE and [177Lu] DOTA-TATE of roendocrine tumors refractory to conventional therapy: preliminary results. Q J Nucl Med Mol Imaging 54: 84-91.

6. Wehrmann C (2007) Results of individual patient dosimetry in peptide receptor radionuclide therapy with 177Lu DOTA-TATE and 177Lu DOTANOC. Cancer Biother Radiopharm 22(3): 406-416.

7. Lawrence $\mathrm{T}$ (2009) Clearance Kinetics and External Dosimetry of Iodine-131-labeled Murine and Humanized Monoclonal Antibody A33 in Patients with Colon Cancer: Radiation Safety Implications. Health Phys. May 96(5): 550-557.

8. Barrington SF (1996) Radiation does rates from patients receiving iodine-131 therapy for carcinoma of the thyroid. Eur J Nucl Med 23: 123-130.

9. Barrington SF (1999) Radiation exposure of the families of outpatients treated with radioiodine (iodine-131) for hyperthyroidism. Eur J Nucl Med 26: 686-692.

10. Guerriero F (2013) Kidney Dosimetry in 177Lu and 90Y Peptide Receptor Radionuclide Therapy: Influence of Image Timing, TimeActivity Integration Method, and Risk Factors. Bio Med Research International pp. 935351.

11. Sandström M (2013) Individualized dosimetry of kidney and bone marrow in patients undergoing 177Lu-DOTA-octreotate treatment. J Nucl Med 54: 33-41. 
12. Kwekkeboom DJ (2008) Treatment with the radiolabeled somatostatin analog [177 Lu-DOTA 0, Tyr3] octreotate: toxicity, efficacy, and survival. J Clin Oncol: Off J Am Soc Clin Oncol 26(13): 2124-2130.

13. Bodei L, Mueller-Brand J, Baum RP (2013) The joint IAEA, EANM, and SNMMI practical guidance on peptide receptor radionuclide therapy (PRRNT) in neuroendocrine tumors . Eur J Nucl Med Mol Imaging 40: 800-816.

14. M Culver, HJ (1991) Dworkin Radiation safety consideration for postiodine-131 hyperthyroid therapy. Nucl Med 32: 169-173.

15. Barrington SF (1999) Radiation exposure of the families of outpatients treated with radioiodine (iodine-131) for hyperthyroidism. Eur J Nucl Med 26: 686-692.

16. Svensson J (2015) Renal function affects absorbed dose to the kidneys and hematological toxicity during 177Lu-DOTATATE treatment. Eur J Nucl Med Mol Imaging 42: 947-955.

17. Garkavij M (2010) 177Lu-[DOTA0,Tyr3] Octreotate therapy in patients with disseminated neuroendocrine tumors: analysis of dosimetry with impact on future therapeutic strategy. Cancer 116: 1084- 1092.

18. Sandström M (2010) Individualized dosimetry in patients undergoing therapy with 177Lu-DOTA-D-Phe(1)-Tyr(3)- octreotate. Eur J Nucl Med Mol Imaging 37: 212-225.

19. Garske U, Sandstrom M, Johansson S (2012) Minor changes in effective half-life during fractionated 177Lu-Octreotate therapy. Acta Oncol 51: 86-96.

20. Larsson M, Bernhardt P, Svensson JB, Wangberg B, Ahlman H (2012) Forssell-Aronsson E. Estimation of absorbed dose to the kidneys in patients after treatment with 177Lu-octreotate: comparison between methods based on planar scintigraphy. EJNMMI Res 2: 49.

21. Baechler S, Hobbs RF, Boubaker A (2012) Three-dimensional radiobiological dosimetry of kidneys for treatment planning in peptide receptor radionuclide therapy. Med Phys 39: 6118-6128.

22. Esser JP (2006) Comparison of [177Lu-DOTA0, Tyr3] octreotate and [177Lu-DOTA0,Tyr3]octreotide: which peptide is preferable for PRRT? Eur J Nucl Med Mol Imaging 33: 1346-1351.

23. Richard Wendt, Janine Wong, Kimberly Selting, Jimmy Lattimer, Jaime (2015) Radiation safety restrictions in the radionuclide treatment of canine osteosarcoma. J Nucl Med May 56(3): 1226.

24. Siegel JA (1999) MIRD pamphlet no. 16: techniques for quantitative radiopharmaceutical biodistribution data acquisition and analysis for use in human radiation dose estimates. J Nucl Med 40: 37S-61S.

25. Heikkonen J (2016) Effect of calculation method on kidney dosimetry in Lu-177-octreotate treatment. Acta Oncol 55: 1069-1076.

26. Miederer M (2012) One single-time-point kidney uptake from Octreo Scan correlates with number of desintegrations measured over 72 hours and calculated for the 6.7 hours halflife nuclide 177Lu. Clin Nucl Med 37: $245-248$.

27. Fernandez R (2015) Ensuring Safe \& Effective Delivery of Lutetium-177 Dotatate Therapy. J Nucl Med 56(S3): 479.

28. J Archer, M Carroll, S Vinjamuri (2014) Clearance of 177Lu-DOTATATE from patients receiving peptide receptor radionuclide therapy Experiences at the Royal Liverpool University Hospital. RAD Magazine 39: 455: 13-15.

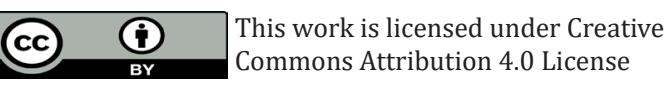

Submission Link: http://biomedres.us/submit-manuscript.php

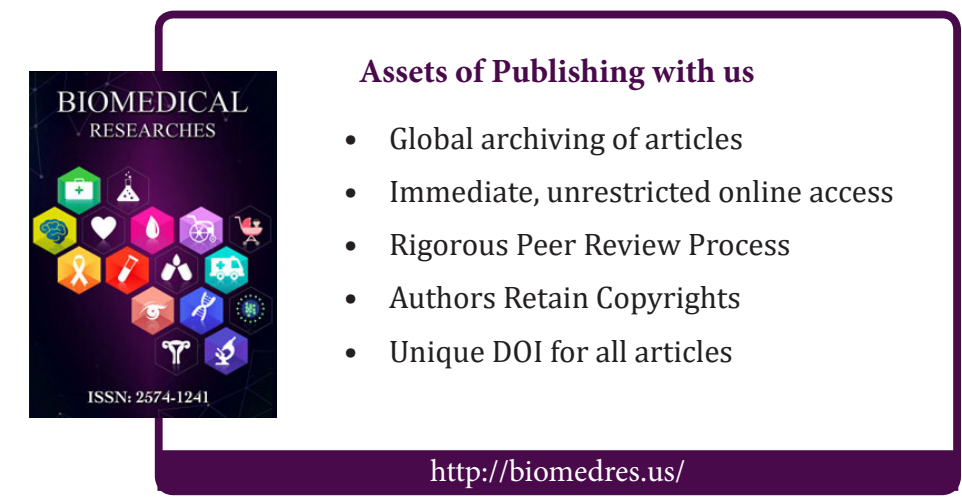

